CARDIOVASCULAR MEDICINE

\title{
Acute and chronic effects of oestradiol on left ventricular diastolic function in hypertensive postmenopausal women with left ventricular diastolic dysfunction
}

\author{
I N Alecrin, J M Aldrighi, M A Caldas, O C E Gebara, N H M Lopes, J A F Ramires
}

Heart 2004;90:777-781. doi: 10.1136/hrt.2003.016493

See end of article for authors' affiliations

Correspondence to:

Dr I N Alecrin, InCor

Secretaria MASS, Av

Eneas de Carvalho Aguiar

44, Cerqueira Cesar, São

Paulo/SP 05403-000,

Brazil; inalecrin@

vol.com.br

Accepted 4 December 2003
Background: Left ventricular function changes in the postmenopausal state. However, there are conflicting reports about the effects of oestrogen on left ventricular diastolic function in postmenopausal women.

Objective: To evaluate the acute and chronic effects of oestradiol in physiological doses on diastolic heart function in hypertensive postmenopausal women with left ventricular diastolic dysfunction.

Methods: A prospective, randomised, double blind, placebo controlled study was used to evaluate the effects of oestradiol in 34 hypertensive women with left ventricular dysfunction. The acute effects of a single $1 \mathrm{mg}$ oral dose of oestradiol or placebo were determined after 90 minutes. The chronic effects of $1 \mathrm{mg}$ oestradiol orally/day or placebo were determined after 12 weeks. Diastolic functional indices (mitral flow and pulmonary venous flow) were assessed by Doppler echocardiography.

Results: Though an appropriate serum concentration was achieved, no acute effect of oestradiol administration on left ventricular diastolic function was identified. After 12 weeks of treatment the following changes (mean (SD)) were identified in the oestradiol group: a decrease in isovolumic relaxation time from $127(23)$ to $106(16) \mathrm{ms}(\mathrm{p}<0.001)$, and in the deceleration time of the mitral $E$ wave from 260 (42) to $238(20) \mathrm{ms}(\mathrm{p}<0.05)$; and an increase in the E/A ratio from $0.8(0.2)$ (basal) to $1.0(0.2)$ after 12 weeks $(p<0.001)$.

Conclusions: Hypertensive postmenopausal women who had hormone replacement therapy over a period of 12 weeks had significant improvement in left ventricular diastolic function. No changes were identified following acute administration.
$\mathrm{P}$ remenopausal women have a lower risk of coronary heart disease than age matched men, whereas after the menopause, when ovarian hormone deficiency becomes evident, the incidence of coronary artery disease accelerates more rapidly in women than in men. ${ }^{12}$ Hormone replacement therapy (HRT) has often been used for relief of symptoms related to the menopause, for the prevention and treatment of osteoporosis, and for its possible cardiovascular benefit. ${ }^{34}$

However, despite extensive observational evidence that oestrogens protect against coronary heart disease, recent controlled clinical studies have failed to show a beneficial effect of HRT on cardiovascular prognosis, and have suggested that it may even increase cardiovascular events. ${ }^{5}{ }^{6}$ On this basis, the use HRT in the treatment and prevention of coronary artery disease has been discouraged. Despite these conflicting results, many women may still be candidates for treatment with oestrogen alone or in combination with progestins to control menopausal symptoms and osteoporosis.

Oestrogen has potential cardioprotective effects through its actions on the lipid profile, vascular reactivity, haemodynamic variables, and fibrinolytic potential. ${ }^{7-9}$ However, the possible cardioprotective role of oestradiol alone has not been well studied in postmenopausal women.

The aging process is associated with cardiovascular abnormalities. The incidence of diastolic functional abnormalities is age related, and heart failure caused by diastolic dysfunction increases dramatically with age, becoming more prevalent in women than men. ${ }^{10}$ Diastolic dysfunction is also associated with coronary heart disease, hypertension, hypertrophic and dilated cardiomyopathy, and valvar disease, and it precedes systolic dysfunction in most cases. Left ventricular dysfunction, whether from aging or from underlying disease, is a strong predictor of mortality. ${ }^{11-13}$
It has been suggested in uncontrolled studies that oestrogen replacement improves systolic and diastolic function and ventricular wall thickness within weeks of starting treatment. ${ }^{914}{ }^{15}$ However, there are conflicting reports about the effects of oestrogen on left ventricular diastolic function in postmenopausal women.

To our knowledge, there has been no study examining the effect of physiological oestrogen replacement on cardiac function. Our aim in this study was therefore to evaluate the acute and chronic effects of physiological doses of oestradiol on left ventricular diastolic function in hypertensive postmenopausal women with documented diastolic dysfunction.

\section{METHODS \\ Subjects}

Nine hundred hypertensive postmenopausal women, aged between 40-65 years, were evaluated between January 1998 and December 2001 in the Heart Institute (InCor) of the University of São Paulo (HC-FMUSP). Among these, there were 40 with left ventricular diastolic dysfunction and no previous HRT use who were eligible for this study.

The postmenopausal state was defined as the presence of amenorrhoea for at least 12 months, or a surgically induced

Abbreviations: A, peak velocity of late diastolic filling; ASE, American Society of Echocardiography; D, peak velocity of diastolic pulmonary venous flow; DTE, deceleration time of the $E$ wave; $E$, peak velocity of early diastolic filling; FSH, follicle stimulating hormone; HERS, heart and estrogen/progestin replacement study; HRT, hormone replacement therapy; IVRT, isovolumic relaxation time; JNC, Joint National

Committee on prevention, detection, evaluation, and treatment of high blood pressure; $S$, peak velocity of systolic pulmonary venous flow; vel a, atrial flow reversal; Vel A, peak velocity of mitral A wave; Vel E, peak velocity of mitral $E$ wave; $W H I$, women's health initiative 
menopause, associated with serum follicle stimulating hormone (FSH) concentrations of $>40 \mathrm{mIU} / \mathrm{ml}$ and serum oestradiol $<20 \mathrm{pg} / \mathrm{ml} .{ }^{16}$ Arterial hypertension was classified by the JNC VI criteria. ${ }^{17}$ The patients included in this study had hypertension stage 2 or 3 .

All subjects underwent routine physical and gynaecological examination. Blood tests, ECG exercise testing, and Doppler echocardiography were done. A Papanicolaou smear, pelvic and transvaginal ultrasonography, and mammography were all normal.

Exclusion criteria were: proven or suspected coronary artery disease; arrhythmias; heart failure functional class $\geqslant 3$; valvar disease of moderate or severe degree; left ventricular systolic dysfunction; thyroid dysfunction; renal or hepatic failure; current smoking; use of selective oestrogen receptor modulators or digoxin; and gynaecological cancers or another known contraindication to oestrogen replacement therapy. Of the 40 women evaluated, two did not have confirmed left ventricular diastolic dysfunction, one had systolic dysfunction, one had had moderate aortic regurgitation, and two had a contraindication to HRT, leaving a final study cohort of 34 women.

The study was undertaken after approval by the ethics committee of the FMUSP, and informed written consent was signed by all the patients.

\section{Study protocol}

The study was a prospective, randomised, double blind, placebo controlled trial. The 34 women formed two groups: 17 treated with oestradiol and 17 with placebo. Demographic and clinical characteristics of the two groups are given in table 1.

A complete cross sectional and Doppler echocardiographic examination, blood hormone concentrations, and systemic blood pressure measurement were done before initiating HRT, 90 minutes after a single dose of either oestradiol or placebo, and 12 weeks after daily dosage of the same treatment.

First, we evaluated the acute effects of a single $1 \mathrm{mg}$ oral dose of oestradiol on left ventricular diastolic function. At baseline, a blood sample was drawn for oestradiol, FSH, and luteinising hormone ( $\mathrm{LH})$ determinations, resting systemic blood pressure was measured, and an echocardiographic evaluation was carried out (ECHO 1). The women were then randomised to receive a single oral dose of either oestradiol or placebo. After a 90 minute rest period, there was a second echocardiographic examination (ECHO 2), and the other measurements were repeated.
To evaluate the chronic effects of HRT, the women continued to receive a daily oral dose of either $\mathrm{l} \mathrm{mg}$ oestradiol or placebo for 12 weeks, at the end of which the echocardiographic measurements (ECHO 3) and the other investigations were carried out for a third time.

All personnel involved in the study were blind to the type of treatment given.

\section{Echocardiographic evaluation}

All echocardiographic examinations were undertaken after 20-30 minutes of rest with the patient breathing quietly and lying in the partial left decubitus position, using Philips-ATL HDI 3000 or 5000 echocardiographic equipment (Advanced Technology Laboratories, Bothell, Washington, USA), with a 3-2 MHz or 4-2 MHz transducer. The findings were recorded on videotape.

M mode echocardiography of the left ventricle was carried out under two dimensional control. The left ventricular internal dimension and interventricular septal and posterior wall thickness were measured at end diastole and end systole, following the American Society of Echocardiography (ASE) recommendation. ${ }^{18}{ }^{19}$ Left ventricular mass was calculated according to the Devereux Penn convention. ${ }^{20}$ Normal left ventricular mass in women was defined as $<96 \mathrm{~g} / \mathrm{m}^{2}{ }^{21}$ Left ventricular systolic dysfunction was considered to be present when the ejection fraction was less than $50 \%$.

Pulsed Doppler measurements of left ventricular diastolic inflow were obtained as reported previously. ${ }^{15} 22$ Briefly, the left ventricular diastolic filling pattern was recorded from the apical position with the sample volume situated between the mitral leaflet tips. The peak velocities of early (E) and late (A) diastolic filling, their ratio (E/A), and the deceleration time of the E wave (DTE) were measured, following the ASE recommendations. The isovolumic relaxation time (IVRT) was measured from the closure spike of the aortic valve to the onset of mitral inflow. Pulmonary venous flow was also measured from the apical four chamber view, and colour Doppler imaging was used to obtain a beam direction as parallel as possible to the direction of the right or left upper pulmonary vein flow. Peak velocities of systolic (S) and diastolic (D) pulmonary venous flow, S/D ratio, and peak velocity of atrial flow reversal (vel a) were measured.

Diastolic dysfunction was defined when the following were present: abnormal left ventricular relaxation $(\mathrm{E} / \mathrm{A}$ ratio $<1$, DTE $>220 \mathrm{~ms}$, and IVRT $>100 \mathrm{~ms}$ ) or pseudonormal filling patterns (E/A ratio of 1-2, DTE of 150-200 ms, IVRT of $60-100 \mathrm{~ms}, \mathrm{~S} / \mathrm{D}$ ratio $<1$, and vel $\mathrm{a} \geqslant 35 \mathrm{~cm} / \mathrm{s}) .{ }^{15}$

All measurements were taken by the same examiner, who was blind to the type of drug given to the patient. Data were

Table 1 Demographic and clinical characteristics of the oestradiol and placebo groups

\begin{tabular}{|c|c|c|c|}
\hline Variable & Oestradiol $(n=17)$ & Placebo $(n=17)$ & p Value \\
\hline Age (years) & $57.7(4.0)$ & $59.5(3.0)$ & NS \\
\hline \multicolumn{4}{|l|}{ Race } \\
\hline White & $14(82.4 \%)$ & $3(17.7 \%)$ & NS \\
\hline Black & $14(82.4 \%)$ & $3(17.7 \%)$ & NS \\
\hline Menopause age (years) & $48.7(4.6)$ & $49.3(2.4)$ & NS \\
\hline Menopause time (years) & $8.59(6.8)$ & $10.18(3.5)$ & NS \\
\hline \multicolumn{4}{|l|}{ Menopause type $\dagger$} \\
\hline Natural & $14(82.4 \%)$ & $12(70.6 \%)$ & NS \\
\hline Surgical & $3(17.7 \%)$ & $5(29.4 \%)$ & NS \\
\hline Diabetes & $4(23.5 \%)$ & $5(29.4 \%)$ & NS \\
\hline \multicolumn{4}{|l|}{ Treatment } \\
\hline ACE inhibitor & $13(76.5 \%)$ & $17(100 \%)$ & NS \\
\hline Diuretic & $14(82.4 \%)$ & $9(52.9 \%)$ & NS \\
\hline$\alpha$-Methyldopa & $3(17.7 \%)$ & $4(23.5 \%)$ & NS \\
\hline Systemic arterial hypertension time (years) & $14(9)$ & $18(6)$ & NS \\
\hline
\end{tabular}

Values are mean (SD) or $\mathrm{n}(\%)$.

$\mathrm{ACE}$, angiotensin converting enzyme. 
Table 2 Doppler echocardiographic diastolic indices of transmitral flow in the oestradiol and placebo groups at baseline, 90 minutes, and 12 weeks

\begin{tabular}{|c|c|c|c|c|}
\hline Variable & Time point & Oestradiol & Placebo & p Value \\
\hline \multirow[t]{3}{*}{ Vel E $(\mathrm{cm} / \mathrm{s})$} & Baseline (T1) & $66(19)$ & $63(11)$ & NS \\
\hline & 90 minutes (T2) & 68 (20) & 61 (13) & NS \\
\hline & 12 weeks (T3) & 74 (22) & 61 (16) & NS \\
\hline \multirow[t]{3}{*}{ Vel A $(\mathrm{cm} / \mathrm{s})$} & Baseline ( $\mathrm{T} 1)$ & 81 (21) & 79 (14) & NS \\
\hline & 90 minutes (T2) & 81 (21) & 76 (11) & NS \\
\hline & 12 weeks (T3) & $75(23)^{*}$ & $73(13)^{*}$ & NS \\
\hline \multirow[t]{3}{*}{ E/A ratio } & Baseline (T1) & $0.8(0.2)$ & $0.8(0.1)$ & NS \\
\hline & 90 minutes (T2) & $0.9(0.2)$ & $0.8(0.1)$ & NS \\
\hline & 12 weeks (T3) & $1.0(0.2) \dagger$ & $0.8(0.2)$ & 0.04 \\
\hline \multirow[t]{3}{*}{ DTE (ms) } & Baseline (TI) & $260(42)$ & $254(22)$ & NS \\
\hline & 90 minutes (T2) & $248(40)$ & $245(20)$ & NS \\
\hline & 12 weeks (T3) & $238(20)^{*}$ & $274(42)^{*}$ & 0.01 \\
\hline \multirow{3}{*}{ IVRT (ms) } & Baseline ( $\mathrm{T} 1$ ) & $127(23)$ & $121(15)$ & NS \\
\hline & 90 minutes (T2) & 121 (17) & 120 (16) & NS \\
\hline & 12 weeks (T3) & 106 (16)† & $121(16)$ & 0.01 \\
\hline \multicolumn{5}{|c|}{$\begin{array}{l}\text { The values are expressed as mean (SD). } \\
\text { * } p<0.05 \text { ( } T 1 v T 3 \text { ) in the same group. } \\
t p<0.001 \text { ( } T 1 v T 3 \text { ) in the same group. } \\
\text { DTE, deceleration time of mitral } E \text { wave; }\end{array}$} \\
\hline
\end{tabular}

the result of averaging three to five different measures on repeated recordings.

\section{Intraobserver variability}

Intraobserver variability of the echocardiographic indices was evaluated in 12 random examinations chosen four months after the first interpretation. The IVRT, DTE, peak velocities of $\mathrm{E}$ and A waves, E/A ratio, peak velocities of $\mathrm{S}$ and $\mathrm{D}$ pulmonary venous flow, and the S/D ratio were analysed.

\section{Statistical analysis}

Quantitative variables are expressed as mean (SD), and qualitative variables in absolute (n) and relative (\%) frequencies. The echocardiographic findings on placebo or oestradiol treatment were compared using the StudentNewman-Keul test. To evaluate an association between two qualitative variables the $\chi^{2}$ or Fisher's test was used.

Evaluation of the group effects (placebo or oestradiol) and the time points of the study (basal, 90 minutes, and 12 weeks) for each of the variables analysed was undertaken by repeated measures analysis of variance (ANOVA) or the Friedman test. The statistical analyses were done using SPSS/ PC+ software, and the level of significance was set at $\mathrm{p}<0.05$.

\section{RESULTS \\ Effect of oestradiol on haemodynamic variables and hormone outcome}

There were no significant differences in heart rate between the oestradiol or placebo groups at any of the time points studied. However, the heart rate slowed significantly after 90 minutes and 12 weeks of treatment in both groups (data not shown). There were also no significant effects on blood pressure or body mass index (BMI) in either group at any of the time points: mean (SD) systolic arterial blood pressure was 153 (22), 150 (17), and 149 (16) $\mathrm{mm} \mathrm{Hg}$ at baseline, 90 minutes, and 12 weeks, respectively, in the oestradiol group, and 149 (16), 152 (15), and 149 (14) $\mathrm{mm} \mathrm{Hg}$ in the placebo group; mean diastolic pressure was 95 (12), 91 (10), and 93 (9) $\mathrm{mm} \mathrm{Hg}$ in the oestradiol group, and 93 (10), 94 (9), and 94 (10) $\mathrm{mm} \mathrm{Hg}$ in the placebo group; mean BMI was 28.9 (4), $28.9(4)$, and $29.0(4) \mathrm{kg} / \mathrm{m}^{2}$ in the oestradiol group, and $28.0(4), 28.0(4)$, and $28.0(4) \mathrm{kg} / \mathrm{m}^{2}$ in the placebo group.

The oestradiol group had a significant decrease in FSH and LH concentrations, from 67 (19) pmol/l and 20 (9) pmol/l at baseline, respectively, to 33 (19) pmol/l and 12 (7) pmol/l after 12 weeks of treatment ( $p<0.001$ for both). FSH and $\mathrm{LH}$ values were unchanged on placebo. At baseline the oestradiol concentration was similar in the two groups. As expected, there were significant increases in the plasma oestradiol concentrations in the treated group, from 20 (0) $\mathrm{pg} / \mathrm{ml}$ at baseline to $85(50) \mathrm{pg} / \mathrm{ml}$ after 90 minutes, and $120(74) \mathrm{pg} / \mathrm{ml}$ after 12 weeks; in the placebo group the concentrations were unchanged over this time.

\section{Effect of oestradiol on Doppler indices}

Basal values of peak velocity of mitral E wave (Vel E), peak velocity of mitral A wave (Vel A), E/A ratio, DTE, and IVRT were not significantly different between the oestradiol and placebo groups. Values of all Doppler indices remained unchanged after 90 minutes of treatment in both groups.

Significant changes resulting from oestradiol treatment were as follows: Vel A decreased in both groups at 12 weeks (but with no significant difference between the groups); DTE was reduced in the oestradiol group after 12 weeks of treatment, and increased in the placebo group; the E/A ratio in the oestradiol group increased after 12 weeks, with no change in the placebo group; and IVRT decreased in the oestradiol group after 12 weeks, to values below those in the placebo group (table 2).

There were no significant differences in pulmonary venous flow indices between the groups at any of the three time points (table 3 ).

\section{Intraobserver variability}

There were significant linear association between the echocardiographic measurements. Pearson's correlation coefficients for the E/A ratio, DTE, IVRT, and S/D ratio were $r=0.909,0.981,0.977$, and 0.966 , respectively $(\mathrm{p}<0.001$ for all). The coefficients of variation of the E/A ratio, DTE, IVRT, and S/D were of $0.072,15 \mathrm{~ms}, 6.27 \mathrm{~ms}$, and 0.12 , respectively ( $\mathrm{p}<0.001$ for all).

\section{DISCUSSION}

To our knowledge, this is the first study to investigate the acute and chronic effects of physiological doses of oestradiol on left ventricular diastolic function in hypertensive postmenopausal women. We found no significant acute effect of a single dose of $1 \mathrm{mg}$ oestradiol on indices of impaired left ventricular diastolic filling, despite achieving an appropriate serum concentration of oestradiol. On the other hand, a significant improvement in diastolic function was observed 
Table 3 Doppler echocardiographic diastolic variables of pulmonary venous flow, left ventricular mass index per unit body surface area, and size of left atrium in the oestradiol and placebo groups at the three time points

\begin{tabular}{|c|c|c|c|c|}
\hline Variable & Time point & Oestradiol & Placebo & p Value \\
\hline \multirow[t]{3}{*}{$\mathrm{S}(\mathrm{cm} / \mathrm{s})$} & Baseline (T1) & $64(16)$ & 59 (12) & NS \\
\hline & 90 minutes (T2) & 61 (13) & 59 (11) & NS \\
\hline & 12 weeks (T3) & 61 (10) & $60(7)$ & NS \\
\hline \multirow[t]{3}{*}{$\mathrm{D}(\mathrm{cm} / \mathrm{s})$} & Baseline (T1) & 41 (10) & 37 (7) & NS \\
\hline & 90 minutes (T2) & $39(8)$ & $37(6)$ & NS \\
\hline & 12 weeks (T3) & $40(8)$ & $41(12)$ & NS \\
\hline \multirow[t]{3}{*}{$S / D$ ratio } & Baseline (T1) & $1.6(0.3)$ & $1.6(0.2)$ & NS \\
\hline & 90 minutes (T2) & $1.6(0.3)$ & $1.6(0.2)$ & NS \\
\hline & 12 weeks (T3) & $1.5(0.2)$ & $1.5(0.2)$ & NS \\
\hline \multirow[t]{3}{*}{ Vel a $(\mathrm{cm} / \mathrm{s})$} & Baseline (T1) & $31(4)$ & $30(3)$ & NS \\
\hline & 90 minutes (T2) & $29(3)$ * & $28(4)^{*}$ & NS \\
\hline & 12 weeks (T3) & $27(3) \dagger$ & $29(2) \dagger$ & NS \\
\hline \multirow[t]{3}{*}{ LVM/BSA $\left(\mathrm{g} / \mathrm{m}^{2}\right)$} & Baseline (T1) & $113(29)$ & $107(28)$ & NS \\
\hline & 90 minutes (T2) & $109(29)$ & $108(26)$ & NS \\
\hline & 12 weeks (T3) & $103(20)$ & $113(28)$ & NS \\
\hline \multirow[t]{3}{*}{ LA size $(\mathrm{cm})$} & Baseline (T1) & $3.7(0.4)$ & $3.6(0.4)$ & NS \\
\hline & 90 minutes (T2) & $3.7(0.4)$ & $3.6(0.4)$ & NS \\
\hline & 12 weeks (T3) & $3.7(0.4)$ & $3.6(0.3)$ & NS \\
\hline \multicolumn{5}{|c|}{$\begin{array}{l}\text { The values are expressed as mean (SD). } \\
\text { * } p=0.02(T 1 v T 2) \text { in the same group. } \\
t p=0.001(T 1 v T 3) \text { in the same group. } \\
D \text {, peak velocity of pulmonary diastolic wave; LA, left atrium; LVM/BSA, left ventricular mass indexed by body } \\
\text { surface area; } S \text {, peak velocity of pulmonary systolic wave; } S / D \text { ratio, the ratio between the peak velocity of } \\
\text { pulmonary } S \text { and D wave; Vel a, peak velocity of pulmonary a wave. }\end{array}$} \\
\hline
\end{tabular}

after 12 weeks of oestradiol replacement, with a reduction in IVRT and DTE and an increase in the E/A ratio. Thus our results show that oestradiol alone produces a significant improvement in diastolic ventricular function in postmenopausal women with hypertension and impaired left ventricular diastolic filling.

Left ventricular diastolic function seems to be affected in the postmenopausal state. For example, postmenopausal women have greater left ventricular wall thickness and lower peak E velocities and E/A ratios on Doppler echocardiography than premenopausal women. ${ }^{9}{ }^{14}$ Moreover, diastolic dysfunction is common in patients with hypertension and also seems to deteriorate with age. ${ }^{13}$

Doppler filling indices are highly dependent on conditions such as age, loading status, heart rate, inotropic state, left atrial size, and ventricular pressure. ${ }^{15} 1^{12-23}$ At baseline in our study, the two groups of women were homogeneously distributed with regard to age, body mass index, left ventricular mass index, and left atrial size, and these indices remained unchanged during the period of study. Heart rate was reduced after 12 weeks of treatment in both groups, but without significant differences between the groups. Our results thus show that the improvement in diastolic function observed after 12 weeks of oestradiol replacement was independent of heart rate, body mass index, left ventricular mass index, or left atrial size.

We also found no differences in the length of time over which the hypertension had been present, the degree of left ventricle hypertrophy, or antihypertensive drug use in the two groups, ruling out the possibility that these variables could have influenced the Doppler indices of left ventricle filling. Moreover, oestradiol did not affect either systolic or diastolic blood pressure, supporting previous work showing that HRT may not be harmful in hypertensive postmenopausal women. ${ }^{24}$

The role of HRT in diastolic dysfunction remains unclear. Recent studies have shown conflicting results, but these discrepancies may be explained by differences in methodology, biological variables, and the types of hormone preparations used. ${ }^{25-28}$
Consistent with our findings, Voutilainen and colleagues showed that chronic HRT improved indices of diastolic flow in healthy postmenopausal women in comparison with a control group not taking HRT, ${ }^{27}$ while no differences in the left ventricular mass, systemic blood pressure, heart rate, or body mass index were found between the groups. However, that study differed from ours in that it was non-randomised, the serum oestradiol levels were not assessed, several different oral preparations were employed, and pretreatment Doppler values were not reported. Snabes and colleagues, on the other hand, found no changes in systolic or diastolic echocardiographic variables, heart rate, or left ventricular mass in normotensive postmenopausal women. ${ }^{26}$ Their results were not surprising as they were analysing healthy women with normal cardiac function at baseline.

Fak and colleagues used a single dose of oral oestrogen and found a significant improvement in left ventricular diastolic filling in hypertensive postmenopausal women with diastolic dysfunction, in contrast to our 90 minute results. ${ }^{28}$ This discrepancy might be explained by the fact that they used a higher dose of conjugated equine oestrogen in their study. Furthermore, only IVRT was shown to be improved, and not the other variables.

We wish to emphasise that our study evaluated the effects of a natural oestrogen-oestradiol—at a physiological dose, in contrast to conjugated equine oestrogen which contains several subtypes of equine steroids with important differences in their pharmacological properties. ${ }^{3}$

Several studies have already shown that Doppler echocardiography has good sensitivity and specificity when analysing transvalvar mitral flow and flow in the lung veins. ${ }^{13} 15222329$ Among four different patterns of left ventricular relaxation identified, the "pseudonormal" pattern in particular can simulate an improvement in left ventricular diastolic function. In our study, we observed two patients in the oestradiol group who presented with this pattern at baseline, and in whom the diastolic filling indices improved after treatment. As most studies have assessed diastolic function only through analyses of transvalvar mitral flow without examining lung vein flow, we suggest that the negative results may reflect 
failure to identify whether diastolic function is normal or pseudonormal.

The use of HRT to reduce cardiovascular risk in postmenopausal women has been questioned since the results of the HERS and WHI studies were published. These appeared widely divergent from the results of earlier observational studies. ${ }^{56}$ However, the therapeutic regimen used in those studies involved conjugated equine oestrogen, either alone or in combination with medroxyprogesterone, in contrast to most of the observational studies, which involved the use of oestrogen alone. Despite uncertainties in the published evidence, we believe that some postmenopausal women may derive cardiovascular benefit from hormone therapy.

In addition, many women on oestrogen replacement may have hypertension and diastolic dysfunction. In this setting, our work adds information showing that oestrogen improves diastolic function under such circumstances. Our results do not suggest that oestrogen treatment is indicated for hypertensive women with diastolic dysfunction in order to improve their cardiovascular prognosis; we did not test that hypothesis. Currently, on the basis of recommendations made by cardiology societies throughout the world, oestrogen replacement therapy should be short term and for those women who have menopausal symptoms. Nevertheless, the ability of oestradiol to improve diastolic dysfunction in hypertensive postmenopausal women during a 12 week course of treatment cannot be dismissed. We believe that oestradiol in physiological dosage may be of value in women with clinical conditions where diastolic dysfunction plays a major role and who also need HRT for symptoms of ovarian insufficiency.

\section{ACKNOWLEDGEMENTS}

This study was supported by a research grant from the Zerbini Foundation, SP, Brazil.

\section{Authors' affiliations}

I N Alecrin, J M Aldrighi, M A Caldas, O C E Gebara, N H M Lopes, J A F Ramires, Heart Institute (InCor), University of São Paulo Medical School, São Paulo, Brazil

\section{REFERENCES}

1 Steingart RM, Packer M, Hamm P, et al. Sex differences in the management of coronary artery disease. The survival and ventricular enlargement investigators. N Engl J Med 1991;325:226-30.

2 Stampfer MJ, Colditz GA, Willett WC, et al. Postmenopausal estrogen therapy and cardiovascular disease. N Engl J Med 1991;325:756-62.

3 Grady D, Rubin S, Petitti D. Hormone therapy to prevent disease and prolong life in postmenopausal women. Ann Intern Med 1992;117:1016-37.

4 Huppert LC. Hormonal replacement therapy: benefits, risks, doses. Med Clin North Am 1987;71:23-39.

5 Hulley S, Grady D, Bush T, et al. Randomized trial of estrogen plus progestin for secondary prevention of coronary heart disease in postmenopausal women - for the heart and estrogen/progestin replacement study (HERS) research group. JAMA 1998;280:605-13.

6 Women's Health Initiative. Risks and benefits of estrogen plus progestin in health postmenopausal women. JAMA 2002;288:321-33.
7 Nabulsi AA, Folsom AR, White A, et al. Association of hormone-replacement therapy with various cardiovascular risk factors in postmenopausal women. The atherosclerosis risk in communities study investigators. N Engl J Med 1993;328:1069-75.

8 Volterrani M, Rosano G, Coats A, et al. Estrogen acutely increases peripheral blood flow in postmenopausal women. Am J Med 1995;99:1 19-22.

9 Pines A, Fisman EZ, Levo Y, et al. Menopause-induced changes in left ventricular wall thickness. Am J Cardiol 1993;72:240-1.

10 Thomas JD, Weyman AE. Echocardiographic Doppler evaluation of left ventricular diastolic function: physics and physiology. Circulation 1991;84:977-90.

11 Schillaci G, Pasqualini L, Verdecchia P, et al. Prognostic significance of left ventricular diastolic dysfunction in essential hypertension. J Am Coll Cardiol 2002;39:2005-11.

12 Wachtell K, Bella JN, Rokkedal J, et al. Change in diastolic left ventricular filling after one year of antihypertensive treatment: the losartan intervention for endpoint reduction in hypertension (LIFE) study. Circulation 2002; 105: 1071-6.

13 Zile MR, Brutsaert DL. New concepts in diastolic dysfunction and diastolic heart failure. Part I. Diagnosis, prognosis, and measurements of diastolic function. Circulation 2002;105:1503-8.

14 Kangro T, Henriksen E, Jonason T, et al. Effects of menopause on left ventricular filling in 50-year-old women. Am J Cardiol 1995;76:1093-6.

15 Garcia MJ, Thomas J, Klein AL. New Doppler echocardiographic applications for the study of diastolic function. J Am Coll Cardiol 1998;32:865-72.

16 World Health Organization. Research on the menopause in the 1990. Reports of a WHO scientific group. WHO: Geneva, 1996.

17 Joint National Committee on prevention, detection, evaluation, and treatment of high blood pressure. The sixth report of the Joint National Committee on prevention, detection, evaluation, and treatment of high blood pressure (JNC VI). Arch Intern Med 1997;157:2413-46.

18 Sahn DJ, De Maria A, Kisslo J, et al. The committee on M-mode standardization of the American Society of Echocardiography. Recommendations regarding quantitation in M-mode echocardiography: results of a survey of echocardiographic measurements. Circulation 1978;58:1072-83.

19 Schiller NB, Shah PM, Crawford M, et al. American Society of Echocardiography committee on standards, subcommittee on quantitation of two-dimensional echocardiograms: recommendations for quantitation of the left ventricle by two dimensional echocardiography. J Am Soc Echocardiogr 1989;2:358-67.

20 Devereux RB, Alonso DR, Lutas EM, et al. Echocardiographic assessment of left ventricular hypertrophy: comparison to necropsy findings. Am J Cardiol 1986;57:450-8.

21 Ilercil A, O'Grady MJ, Roman MJ, et al. Reference values for echocardiographic measurements in urban and rural populations of differing ethnicity: the strong heart study. J Am Soc Echocardiogr 2001;14:601-11.

22 Oh JK, Appleton CP, Hatle LK, et al. The noninvasive assessment of left ventricular diastolic function with two-dimensional and Doppler echocardiography. J Am Soc Echocardiogr 1997; 10:246-70.

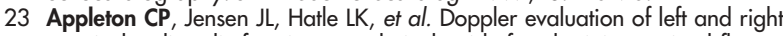
ventricular diastolic function: a technical guide for obtaining optimal flow velocity recordings. J Am Soc Echocardiogr 1997;10:271-97.

24 Sumino H, Ichikawa S, Kumakura $\mathrm{H}$, et al. Effects of hormone replacement therapy on office and ambulatory blood pressure in Japanese hypertensive postmenopausal women. Hypertens Res 2003;26:369-76.

25 Sites CK, Tischler MD, Blackman JA, et al. Effect of short-term hormone replacement therapy on left ventricular mass and contractile function. Fertil Steril 1999;71:137-43.

26 Snabes MC, Payne JP, Kopelen HA, et al. Physiologic estradiol replacement therapy and cardiac structure and function in normal postmenopausal women: a randomized, double-blind, placebo-controlled, crossover trial. Obstet Gynecol 1997;89:332-9.

27 Voutilainen S, Hippelainen M, Hulkko S, et al. Left ventricular diastolic function by Doppler echocardiography in relation to hormonal replacement therapy in health postmenopausal women. Am J Cardiol 1993:71:614-17.

28 Fak AS, Erenus $M$, Tezcan $H$, et al. Effects of single dose of oral estrogen on left ventricular diastolic function in hypertensive postmenopausal women with diastolic dysfunction. Fertil Steril 2000;73:66-71

29 Spirito P, Maron BJ, Bonow RO. Noninvasive assessment of left ventricular diastolic function: comparative analysis of Doppler echocardiographic and radionuclide angiographic techniques. J Am Coll Cardiol 1986;7:518-26. 